\title{
The Big Data toolkit for Psychologists: Data Sources and Methodologies
}

\author{
Heinrich Peters, Zachariah Marrero, Samuel D. Gosling
}

As human interactions have shifted to virtual spaces and as sensing systems have become more affordable, an increasing share of peoples' everyday lives can be captured in real time. The availability of such fine-grained behavioral data from billions of people has the potential to enable great leaps in our understanding of human behavior. However, such data also pose challenges to engineers and behavioral scientists alike, requiring a specialized set of tools and methodologies to generate psychologically relevant insights.

In particular, researchers may need to utilize machine learning techniques to extract information from unstructured or semi-structured data, reduce high-dimensional data to a smaller number of variables, and efficiently deal with extremely large sample sizes. Such procedures can be computationally expensive, requiring researchers to balance computation time with processing power and memory capacity. Whereas modelling procedures on small datasets will usually take mere moments to execute, applying modeling procedures to big data can take much longer with typical execution times spanning hours, days, or even weeks depending on the complexity of the problem and the resources available. Seemingly subtle decisions regarding preprocessing and analytic strategy can end up having a huge impact on the viability of executing analyses within a reasonable timeframe. Consequently, researchers must anticipate potential pitfalls regarding the interplay of their analytic strategy with memory and computational constraints.

Many researchers who are interested in using "big data" report having problems learning about new analytic methods or software, finding collaborators with the right skills and knowledge, and getting access to commercial or proprietary data for their research (Metzler et al. 2016). This chapter aims to serve as a practical introduction for psychologists who want to use large datasets and datasets from non-traditional data sources in their research (i.e., data not generated in the lab or through conventional surveys). First, we discuss the concept of big data and review some of the theoretical challenges and opportunities that arise with the availability of ever larger amounts of data. Second, we discuss practical implications and best practices with respect to data collection, data storage, data processing, and data modelling for psychological research in the age of big data. 


\section{What is Big Data?}

The term "big data" is discussed across many scientific disciplines but there is still no single widely accepted definition of the concept. In fact, different disciplines define big data in vastly different ways. In the fields of computer science and engineering, big data is usually defined as data that is generated with such volume, variety, and velocity that special infrastructure and software is needed to store, process, and analyze it (see Borgman, 2015). Companies like Amazon, Google, and Facebook, for example, maintain data centers consisting of hundreds of thousands of servers needed to manage the quantities of data that are generated on their platforms. Another common definition states that datasets that do not fit in the memory of a single machine can be considered big data. Of course, such definitions depend on the tools and technologies that are available in a given field at a given point in time, rendering the concept of "big data" a moving target. Until recently psychologists have rarely had access to quantities of data that pose challenges to state-of-the-art hardware and software, and the term "big data" has commonly been used to describe data from non-traditional data sources, such as social network sites or smartphone apps. Even though the vast majority of such datasets would not be considered "big" in the fields of computer science or engineering, they typically are: 1) much larger than the datasets psychologists have traditionally been generating through lab experiments and surveys, and 2) require and enable analytic techniques that have traditionally not been part of behavioral scientists' repertoires. In other words, when psychologists use the term big data they often refer to data that is "big" relative to the standard of the field, and that mimics "actual" big data in terms of how it is generated, processed, and analyzed.

\section{A Psychological Perspective on Big Data - Big N, Big V, Big T}

Raymond B. Cattell (1988) famously argued that the methods used in psychological research should be informed by the structure of psychological data. Cattell suggested that data can be classified along three dimensions: persons, variables, and occasions. This taxonomy can be leveraged to describe the size and complexity of data sets, with sample size (big $\mathrm{N}$ ), number of variables (big $\mathrm{V})^{1}$, and temporal structure (big T) serving as the defining criteria of big data in psychological research (Adjerid \& Kelley, 2018).

\footnotetext{
${ }^{1}$ In computer science and statistics the number of rows and columns in a matrix are often denoted as $\mathrm{n}$ and $p$, such that $p$ represents the number of variables. We will use this notation in a later section on computational complexity but we will continue to use "big V" when we refer to the taxonomy of big data introduced by Adjerid \& Kelley, 2018.
} 
Big $N$ refers to data from a large number of individuals. In the context of behavioral science this includes sample sizes of hundreds of thousands or millions of individuals. Person-level data collected from social media profiles or through large-scale online surveys usually falls into this category. The high participation rate in digital platforms allows for the collection of diverse samples and gives researchers access to demographics that have been traditionally underrepresented in psychological research (Gosling et al., 2004; Kosinski et al., 2015). Furthermore, large samples allow for the investigation of small, but meaningful effects (Matz et al., 2017) and the use of complex models while avoiding overfitting (Yarkoni \& Westfall, 2017). The latter point is especially important in light of the tendency of underpowered studies to produce not only type II but also type I errors, which may have contributed to the so-called replication crisis (Open Science Collaboration, 2015).

Big $V$ data refers to the number, diversity, and complexity of variables a dataset contains. Big $V$ data is prevalent in exploratory contexts where a multitude of potential predictors is considered; this is often the case when semi-structured or unstructured data are used. Many methods in natural language processing (NLP), for example, rely on the analysis of word-frequency distributions within and across documents. Text data are typically represented in the form of document-term-matrices, where the frequency of each individual word is treated as a variable. Hence, text data are typically very high-dimensional. Similarly, image data are usually high dimensional because a variety of features can be extracted from images. Big $V$ data can also be generated through feature engineering, (i.e., creating large numbers of derived variables from lower dimensional raw data). Big $\vee$ data poses special challenges to statistical procedures (e.g., overfitting, computational costs) and calls for specialized techniques including regularization and variable selection or dimensionality reduction.

Big $T$ refers to data that contains large amounts of temporal information (i.e., a large number of measurement occasions per person). This property is native to studies involving log data or physiological measures (e.g., smartphone sensing or EEG studies), where data can be recorded through a device's sensing systems with near continuous granularity, which can result in hundreds of thousands of measurements per person. Such granularity of observations offers unprecedented opportunities to describe human behavior in great detail and to understand within-person psychological processes. Complex longitudinal data calls for sophisticated aggregation methods in order to derive interpretable insights. Machine learning techniques can be used to cluster or classify events and activities based on their underlying temporal structure. Finally, big $\mathrm{T}$ data may require the use of specialized statistical models for longitudinal data, such as time series regression, growth curve modelling, or time-to-event regression, and specialized preprocessing in order to account for timelags or to ensure stationarity. 
Importantly, data can be 'big' on one, two, or all three dimensions and in practice the boundaries between big $\mathrm{V}$, and big $\mathrm{T}$ may be fuzzy, because a big $\mathrm{T}$ dataset can be turned into a big $\mathrm{V}$ dataset by extracting large numbers of variables, which capture how each individual behaves over time. GPS data, for instance, usually consists of only three main variables (latitude-longitude pairs and time stamps) ${ }^{2}$, with thousands of data points collected per individual; this longitudinal data can be aggregated in numerous ways to create meaningful person-level variables and it can be enriched with additional information about the characteristics of the areas and places visited by each person (e.g., demographics, events, weather, etc.), resulting in an even richer set of variables.

\section{Theoretical considerations: Explanation VS Prediction}

Psychology has traditionally been chiefly concerned with causal explanations of human behavior. Explanatory research uses statistical modelling to test causal hypotheses derived from theory (Shmueli, 2010). The predominant methods of explanatory research in the field of psychology revolve around controlled experiments and statistical inference, where conclusions about the broader population are derived from the analysis of in-sample relationships and models are used to confirm predictions from theory. These procedures follow a linear process that relies on researcher expertise and statistical theory. First, researchers must select variables and forms of association (e.g., interactions, curvature) that are plausibly related to the dependent variable. Second, researchers select an appropriate data model (e.g., linear model), which is explicitly defined in statistical theory (usually frequentist), and estimate its associated model parameters from the sample data. Third, researchers apply statistical inference tests (i.e., significance tests) to the model parameters based on statistical theory and then confirm that the observed test score (e.g., t-score, p-value, F-ratio) is valid by ensuring that the statistical assumptions required by the test have been satisfied. Fourth, if the assumptions are satisfied and the researcher has concluded that there is a low probability that the data could have been generated by chance, the test is interpreted as evidence that the model is generalizable. Alternatively, if the final model did not yield sufficient evidence, researchers are discouraged from repeating the analysis with different analytic decisions on the same data because doing so corrupts the statistical inference (e.g., Type I error inflation). Despite the widespread popularity of the explanatory approach among psychologists, the current replication crisis suggests that existing explanatory models in the field of psychology generalize poorly to new samples (Camerer et al., 2018; Yarkoni \& Westfall, 2017). This may be explained in part by an overreliance on small sample sizes in experimental research and in part by questionable research practices and procedural overfitting (i.e., p-hacking).

${ }^{2}$ GPS records can also contain other variables such as altitude, bearing, and accuracy estimates. 
Predictive research, in contrast to explanatory research, is generally not concerned with causal theory and focuses squarely on the empirical generalizability of statistical models to new or future observations (Shmueli, 2010). For example, whereas the traditional explanatory procedure uses a number of assumptions to conclude that an estimated model is representative of any sample from the population, predictive models are specifically selected based on how well a model estimated in one sample accounts for variation in a different sample. To do this, predictive modeling relies heavily upon algorithmic procedures that are far less demanding on statistical theory (e.g., cross-validation, hyperparamter tuning, feature selection). As in explanatory modelling, researchers must first select the variables of interest, but the theoretical relationships between the variables are usually not a primary concern. Second, researchers have to prepare their data for analysis according to the algorithms they wish to apply. Third, researchers apply the algorithm(s) and evaluate the effectiveness using evaluation metrics that capture how well a model performs on new data. Fourth, a judgment is made regarding the quality of the prediction, usually by comparing the performance against a reference model (e.g., a model predicting the mean in the case of continuous data or the mode in the case of categorical data).

In the past, behavioral and social scientists have largely avoided this purely predictive, less theory driven approach, but the rise of big data with its wealth of unstructured, associative, behavioral data has made predictive research more attractive. The attraction of predictive models is driven by three key factors. First, large real-world datasets often contain complex patterns and relationships that have little grounding in existing theory and are hard to cast in terms of simple hypotheses. Second, the traditional methodological toolkit of psychological scientists is quickly pushed to its limits when confronted with big data. Significance levels lose their meaning when applied to very large samples because even the tiniest effects become significant (big- $\mathrm{N}$ ) and traditional statistical methods are ill equipped to deal with an abundance of predictors (big-V) or complex temporal structures with many measurement instances (big-T). Third, big data enables researchers to employ "data-hungry" machine learning techniques and to test the generalizability of their models "out-of-sample", which constitutes a key aspect of the predictive framework. At the same time, the methods that are most suitable to analyze large, complex datasets, such as shrinkage models (Lasso, Ridge, Elastic Net), ensemble models (Random Forests, Gradient Boosting), or "black-box" models like Neural Networks, are not well suited for explanatory research. Penalized linear models models, for example, produce biased coefficient estimates, and Random Forests, Gradient Boosting, and Neural Networks, capture complex non-linearities and interactions, which are hard or impossible to infer from model parameters and would be too complex to interpret in light of existing theory. 
Nonetheless, as Shmueli (2010) points out, the predictive approach still serves several vital scientific functions: First, by taking into account complex data and a multitude of potential relationships, predictive modelling can uncover new phenomena and causal mechanisms, thereby generating new hypotheses and starting points for theoretical advancement and explanatory approaches. Second, predictive modelling can be used to discover new measures and operationalizations; for example, personality scores extracted from social media data seem to predict a range of outcome variables better than questionnaire-based trait scores (Kulkarni et al., 2018). Third, predictive modelling can serve as a

"reality check" for existing explanatory theories by assessing their predictive power and generalizability in real-world contexts. To leverage the benefits of predictive modelling psychologists must expand their methodological toolkit. The next section will serve as an introduction for psychologists who want to take advantage of non-traditional datasets and engage in predictive research. For an in-depth discussion of explanation and prediction in statistics and psychology, interested readers are directed to Shmueli (2010), Yarkoni and Westfall (2017), and Stachl et al. (2020).

\section{Practical Recommendations and Tools}

The present section offers an introduction to some of the practical aspects of working with large, complex datasets. Given the scope of the present chapter, we can only scratch the surface of the many issues relevant to data collection, storage, processing, and modelling. However, the current section may serve as a starting point for researchers who are planning to work with big data.

\section{Data collection}

Digital platforms and other companies collect an abundance of behavioral data that can be used for psychological research. In addition to collecting their own data, psychologists can gain access to interesting secondary data in multiple ways, including by querying data that is made available by companies through specific application programming interfaces (APIs), scraping unstructured data from the web, or by collaborating with companies to gain access to their user base and data. The present section briefly outlines each of these options.

Application Programming Interfaces (APIs)

An API is a computing interface that allows applications to communicate with each other. As such, APIs allow for the transfer of data between applications and enable the integration of third-party 
applications. For instance, if a user logs into an app with their Facebook or Google account, this process is handled in the background by a Facebook or Google API, which confirms the user's authenticity and gives the app access to some of the user's data in a standardized process. In the context of data collection for psychological research, APIs can be utilized to authenticate and track users across different platforms (given informed consent), and researchers can query the APIs of digital platforms to access data that is made available by the respective provider. Generally, APIs are the official infrastructure that a provider wants third-parties to use. Hence, they should be the first option to consider when data has to be collected from social media platforms or other websites.

The most common type of APIs are representational state transfer (REST) APIs. REST is a standard that defines architectural constraints for web services to ensure interoperability. REST APIs can access and manipulate web resources as identified by their URLs through the Hypertext Transfer Protocol (HTTP). The most important types of HTTP commands enabled by REST APIs are: GET (fetch data), PUT (edit existing data), POST (add new data), and DELETE (delete data). For researchers interested in data collection, GET is the most important command. GET takes as input a Uniform Resource Identifier (URI) consisting of a base URL and a list of query parameters, usually including an authentication key/token.

The response of a REST API call contains the requested data, typically formatted in XML or JSON. REST API calls are facilitated by libraries like $h t t r$ in $\mathrm{R}$ and requests in Python, which allow users to efficiently manage query parameters and extract relevant data from the response. Besides REST APIs there are other APIs, such as the Facebook graph API, which allows third parties to access the network graph representing users, their relationships, and records of their personal data. The graph API can be queried just like a REST API by combining a base URL with a list of query parameters. Prominent research that made use of the Facebook API was published by Kosinski et al. (2013) who collected behavioral data along with self-report data from dozens of psychometric tests from more than 10 million Facebook users (Kosinski et al., 2015) volunteering to donate their data. The same API was later abused by Cambridge Analytica in violation of user agreements to harvest Facebook profiles of millions of users and run targeted political ads (Isaak \& Hanna, 2018; Schneble et al., 2018). In response to the increasing societal awareness of privacy concerns, businesses have become increasingly strict with regard to the data they allow third parties to access through their APIs, which poses challenges for researchers.

Web-Scraping 
The term "web-scraping" describes a range of methods that automatically extract information from websites by transforming the unstructured source data of a web page into a structured format. Taking advantage of regularities in URL structure, web page structure, and content structure, it is often possible to scrape large amounts of data by looping over many web pages. Each web page has a source code written in markup language (e.g., HTML), which contains its information in a machine readable format. Web browsers, for instance, read the source code to allow them to display the content of a webpage to the user. Web scraping typically follows a five-step process to extract information from the source code of web pages: First, it is necessary to locate the URLs of the web pages that contain the relevant data. Second, the source code needs to be fetched from each URL. Third, the source code is parsed into logical units according to its markdown structure (typically HTML elements). Fourth, it is possible to search for the elements that contain the relevant data. And fifth, the often-unstructured data need to be reformatted into a structured format for further analysis. These steps can be automated using loops and other control structures in conjunction with powerful libraries, such as requests and BeautifulSoup in Python, or httr and rvest in R.

Such libraries facilitate the process considerably but web-scraping has several important drawbacks: It is still relatively laborious and error prone, data quality is often less than optimal, and it relies critically on decisions made by third parties. In fact, many online platforms prohibit scraping and protect themselves against it, for example by imposing rate limits on IP addresses, or requiring login credentials. For most legitimate websites, researchers can find out to what extent they are allowed to scrape, by appending "/robots.txt" to the website's base URL. As a rule of thumb: whenever a platform has an API, it is advisable to use this official interface and use scraping only as a last resort.

Industry Collaborations

Large datasets can be collected through research collaborations with companies and other organizations. Many companies collect large amounts of data as a part of their day-to-day business operations. Such secondary data can be of great value for researchers. Alternatively, companies are sometimes willing to participate in the collection of primary data by leveraging their platforms and customer base. For example, relatively large datasets of psychological data have been collected through surveys in collaboration with the British Broadcasting Company (BBC; Rentfrow et al., 2015) and Time Magazine (Ebert et al., 2019). High-profile industry collaborations promise high rewards but there are usually high barriers that prevent companies from sharing proprietary data with researchers. These barriers include privacy and legal concerns resulting from the high level of public scrutiny to which large companies are subjected. However, companies sometimes make data available to the public in 
anonymized and aggregated form in the context of "data for good" initiatives. For example, Facebook made available aggregate mobility data from roughly 29 million users to enable researchers to study the adoption of social distancing during the COVID-19 crisis (https://dataforgood.fb.com). But it is not only tech giants that accumulate data of interest to psychologists; startups often collect substantial amounts of data that could be used for psychological research. It is sometimes easier to collaborate with startups because they tend to impose lower bureaucratic hurdles and usually benefit more from researchers working with their data; for example, collaboration with researchers can lend credence to a company's product or provide management with valuable information about its user base.

\section{Data Storage}

Collecting large amounts of data requires dealing with issues of data storage. Smaller datasets that do not require frequent updates can easily be held as files on local drives but larger, but more complex data require a principled approach to data storage in order to maintain data integrity and to ensure that the data can be easily accessed, updated, and shared; this can be achieved through traditional Relational Database Management Systems (RDBMSs) or distributed systems optimized for big data processing, such as Apache Hadoop or data warehouse solutions like Amazon Redshift or Google BigQuery.

The term RDBMS refers to databases that store data in tables or "relations" consisting of rows and columns. Tables represent real-world entities and their relationships with each other, such that insights can be generated by merging and filtering related tables. For example, one table could contain demographic data for users of an app, while other tables would contain time-stamped behavioral records, indicating every time a user has opened and closed the app or interacted with any feature within the app. A researcher can then calculate derived metrics like overall app-usage time per user, merge this derived table with the demographic data and filter the resulting table to obtain data only from individuals born in a specific geographic region. Every relation in an RDBMS is represented by a set of schemata: A Logical Schema, is a conceptual model of the data, which describes data types and variable names, etc. A Physical Schema describes the data layout (files, indices) and how the data are represented in secondary storage. A Virtual Schema (View) refers to derived tables, which are generated by way of merging and filtering in order to derive insights from data. Most popular RDMBSs use the Structured Query Language (SQL) for querying and maintenance. SQL is a data manipulation language in the sense that it allows users to query, insert, delete, and modify entries, but also a data definition language because it allows users to define relational schemata (i.e., create, alter, and delete tables and their attributes). RMDBSs are useful because they provide a highly reliable, standardized, and intuitive way to organize structured or 
semi-structured data. However, they are quickly stretched to their limits once the volume of data exceeds the realm of gigabytes, especially in the case of unstructured data.

If truly massive amounts of data need to be stored and processed (i.e., data that is too large for a single server), distributed files systems are a viable option. A popular open source file system is Hadoop Distributed File System (HDFS), which organizes large files across multiple machines. Data stored in HDFS can be processed with MapReduce, a programming model that is optimized for parallel and distributed processing. A MapReduce-command is typically composed of a map-function, which filters and sorts entries according to specified criteria, and a reduce function, which performs summary operations. Importantly, HDFS also enables queries in SQL-like syntax through systems like Apache Hive or Apache Spark SQL. Apache Spark additionally contains a library of parallelized, distributed data manipulation commands and distributed machine learning algorithms optimized for big-data applications. These libraries are crucial to make efficient use of distributed storage and computational resources. All of the big-data systems mentioned above have programming interfaces for $\mathrm{R}$ and Python.

RDBMS as well as distributed file systems can be hosted either on private servers or in the cloud (e.g., Amazon Web Services [AWS], Google Cloud Platform [GCP], Microsoft Azure). There are several advantages to using cloud systems: First, cloud platforms provide access to preconfigured infrastructure and environments, so very little effort is required for setup and maintenance, compared to a private server. Second, storage is virtually unlimited and can easily be extended or reduced depending on the user's current needs. Third, if large amounts of data need to be processed and analyzed, this can be done within the cloud environment, using the computational resources of the cloud, without the need to transfer any data. Private servers, on the other hand, can in rare cases offer more fine grained control, and may be required due to privacy protocols (e.g., University ethics guidelines may prevent researchers from storing sensitive data on cloud platforms). Another cloud-based alternative to traditional RDMBs and distributed file systems are data warehousing solutions like Amazon Redshift or Google BigQuery, which mimic the look and feel of traditional SQL query tools, but are optimized for big data and provide an extensive array of additional analytic capabilities.

We have summarized a variety of options for data storage, ranging from local drives to traditional RDBMSs, open source distributed files systems, and commercial data warehousing solutions. The optimal solution for a given research project depends on the scale, access frequency, and update frequency of the data. Typically, large scale projects with high access and update frequency require more principled storage solutions (i.e., RDBMS, distributed file systems, or data warehousing). Finally, researchers do not have to become database engineers but it is certainly advisable to learn the basics of SQL and how to set up and connect to remote databases or distributed file systems. 


\section{Data (pre-) processing}

Data preprocessing is the transformation of raw data into a format that is suitable for modelling. Working with complex data requires a flexible, but principled approach to preprocessing. Big data, like any data that was not collected in a controlled lab setting, poses specific challenges to preprocessing:

1. Raw data often contain a significant amount of low quality data and information that is irrelevant to the research question at hand.

2. Information is usually distributed across many different data sources, which need to be filtered and merged.

3. The data are often very sparse and contain a lot of missing or corrupted values.

4. Psychologically relevant variables need to be derived from fine grained behavioral data and often the level of granularity needs to be reduced in order to answer psychologically relevant questions. To address these challenges, researchers need to make important decisions with regard to filtering and merging data from different data sources, handling missingness and sparsity, as well as feature engineering.

\section{Filtering and Merging}

Big data is often secondary data, which was not originally collected for the purpose of psychological research and may include a lot of information that is irrelevant to the research question at hand. At the same time, the information is usually distributed across many different data sources that need to be combined to answer psychologically relevant questions. As a consequence, researchers need to filter the raw data to extract the relevant information and to merge data to bring together records of the same entities in the appropriate units of analysis. The removal of irrelevant information is a complex process because sophisticated analyses may be needed to classify the data. The merging of data may be challenging because there is not always a unique identifier that allows records to be matched.

Filtering and merging can, in principle, be performed at two stages in the data analysis process: 1) when querying the data from a database or 2) after reading the data into the data analysis environment. If it is already clear which records are required for an analysis and there is also a unique identifier that allows related records to be matched, filtering and merging should be performed early on (i.e., when querying the database). This approach reduces analytic flexibility but it is usually preferable when working with large datasets because it reduces the use of memory and computational resources. 
Performing the filtering and merging in the data analysis environment has the disadvantage of loading irrelevant data into memory only to be discarded later on, which can easily become a problem when large amounts of data are processed. Both $R$ and Python can be used to query RDBMS or HDFS and other cloud systems.

However, it is not always possible to filter the required data at the query stage because doing so often requires a deep understanding of the data and may be based on complex exploratory analyses. For example, when analyzing data from social media platforms, researchers may be interested only in specific types of content, such as posts discussing a certain topic. Filtering content can require the use of classification algorithms as part of the pre-processing procedure. Similarly, it may be necessary to distinguish between activity generated by actual users and activity generated by bots, which would be considered noise in most psychological contexts. An estimated $15 \%$ of Twitter "users" are bots and identifying bots may require sophisticated classification models (Varol et al., 2017).

In many applications a critical component of data quality is the amount of data that is available per person. Especially when raw data are transformed into derived metrics, the measurement quality of the derived metrics depends on the amount of raw data available for each person (e.g., Youyou et al., 2015). In the context of user data, the amount of data generated per user often follows a gamma distribution where many users generate very little data and an increasingly smaller number of users generates a lot of data. For example, many people install an app and never use it or open it just a few times, whereas a much smaller subset of people will use the app regularly over a long period of time. Consequently, derived measures will differ greatly from user to user in terms of quality. It is therefore important to implement sensible exclusion criteria as part of the preprocessing procedure. Setting a low threshold sacrifices measurement quality while preserving sample size. Setting a high threshold can increase measurement quality while reducing sample size, but can bias the data in subtle ways, especially when the absence of data is not just the result of idiosyncrasies in data collection, but is indicative of the absence of behavior. In GPS-tracking studies, for example, people's location trajectories are sometimes recorded though event-based sampling (i.e., a person's location is recorded only if the person moves from one location to the next); by implication, the people who show the least amount of mobility will produce the smallest number of data points while the lack of mobility may be related to key variables (e.g., mental health outcomes; Mueller et al., in Press). Setting a strict threshold on the amount of mobility data may therefore discard an important part of the distribution and decrease the chance of detecting meaningful relationships between mobility and relevant outcome variables.

Merging data can be a challenge, especially when there is no unique identifier across multiple data sources, which is often the case for secondary data collected from multiple sources. If researchers 
have control over data collection the identifiability of records should be a primary concern. For example, it is possible to track users across different platforms by using APIs for authentication or by recording device identifiers. If there is no unique identifier, data can still be merged on the basis of other identifying information such as names, addresses, and birth dates or other demographic data and GPS data. However, such data is rarely formatted identically across data sources. Nonetheless it is possible to merge such data based on fuzzy matching techniques, which take into account the similarity, rather than exact congruence, of one or more identifying variables. The packages fuzzyjoin in $\mathrm{R}$ and fuzzywuzzy in python enable fuzzy merging, but they do not perform very well at scale. For truly large scale datasets in the range of millions of records it is necessary to use more complex techniques, such as the fastlink algorithm (Enamorado et al., 2019), which is implemented in the fastlink package for R. The same algorithm is implemented in the PySpark package splink, which works well at even greater scale (100 million records or more). Fuzzy merging algorithms can also be used for the purpose of deduplicating (i.e., removing duplicated records from a dataset).

\section{Missing and Sparse Data}

Missing data is a prevalent phenomenon in big data contexts. In many cases a substantial proportion of the data is missing, especially when datasets are generated by combining data from multiple sources. The issue of missing data deserves particular attention as missingness can have significant impact on statistical analyses and may be handled differently, depending on the researchers' choice to engage in explanatory or predictive research. Missing data can be categorized into three categories: 1) missing completely at random (MCAR), when missingness depends neither on observed variable values nor on unobserved variable values themselves; 2) missing at random (MAR), when missingness does not depend on the unobserved values themselves, but is explained by observed variable values; and 3) missing not at random (MNAR), when missingness depends on the unobserved values themselves (i.e., the data is neither MCAR nor MAR). The missingness mechanism is important because it can inform researchers' choices with respect to preprocessing and modelling.

Generally, missing data can be addressed in three different ways. Researchers may decide to 1) delete records affected by missingness, 2) impute missing values, or 3) use the fact that a datapoint is missing as additional information in a predictive model. First, deleting records with missing values is the most simple strategy but it may reduce sample size and power; this is usually not a concern in big $\mathrm{N}$ contexts but it can be prohibitive in big $\mathrm{V}$ contexts, where almost all records may have some missing values. Additionally, deletion can lead to biased coefficient estimates in the absence of MCAR. Second, imputation of missing values can be performed by substituting missing values with measures of central 
tendency (e.g., mean) or through model-based and multiple imputation, where missing values are predicted from observed values. Model based imputation can be computationally costly in big data contexts but it leads to more realistic distributions compared to mean substitution. Imputation, especially mean substitution, increases sample size relative to the amount of information that is captured in a dataset, which may bias error estimates and render imputation problematic in the context of explanatory modelling and statistical inference. Third, in certain cases missingness can be highly informative of the target variable (when the data is not MCAR). Therefore the performance of predictive models can sometimes be dramatically improved by including dummy variables indicating missingness. In financial fraud prediction, for example, failure to report certain metrics may be indicative of fraudulent behavior. Similarly, failure to respond to self-report surveys or record one's behaviors may be indicative of the very construct that is being measured, such as when tracking alcohol intake or monitoring depressive symptoms. Explicitly modelling missing data can thus be helpful in predictive research, while it is usually not expedient in explanatory research.

Related but distinct from the issue of missing data is the issue of sparse data. Especially when dealing with behavioral records, it is crucial for researchers to know whether behavior might have occurred but failed to be recorded (missing data), or whether behavior was correctly not recorded because it did not occur (sparse data). Behavioral data is typically captured as a list of time-stamped events, which are transformed into derived metrics. When such a table is merged with other data it is very likely that some records cannot be matched because not every individual exhibited every type of behavior. This assumption is especially true in the context of big data where the data may be composed of a wide variety of different behaviors and includes a large number of individuals with idiosyncratic behavioral profiles. Hence, a combined table will contain a large number of missing values, which actually represent the absence of behavior rather than the failure to record behavior. Such data should never be treated as missing. Instead, researchers have to rely on their knowledge of the data-generating process to fill in the correct values. In most scenarios the absence of behavior will be coded as zero, resulting in data matrices consisting of zeros to a substantial degree. The same is true in many applications involving text analysis and natural language processing techniques, where document-term-matrices are often sparse and consist mostly of zeros. Sparse data poses several unique challenges to pre-processing and modelling. For example, both $\mathrm{R}$ and Python support a special data format for sparse matrices, which is much more efficient in terms of memory and computation than are matrices containing explicit zeros, especially when it comes to larger datasets. Additionally, some of the routine pre-processing steps may have unintended consequences when applied to sparse feature matrices. For example, it is usually not advisable to normalize sparse data because it turns zero-cells into non-zero cells, thereby creating a dense matrix, which can dramatically increase memory usage. Similarly, some implementations of Principal Components Analysis (PCA) center the input data before computing the singular value 
decomposition (SVD). In such cases it is recommended to use alternatives such as truncated SVD, also known as latent semantic analysis (LSA). Sparse target variables may also require specific statistical techniques, such as zero-inflated models.

\section{Feature engineering}

Depending on the structure of the data, a significant proportion of effort has to be devoted to feature engineering. Feature engineering describes the process of transforming raw data into meaningful metrics, which can be used as predictors in a model. Psychological constructs have traditionally been measured through questionnaires such that variables can easily be generated by summing up individual item responses. This is not possible in the case of complex multivariate and longitudinal behavioral data. The most common feature engineering tasks in psychological research consist of 1) deriving psychologically relevant measures from very granular, semi-structured, or unstructured data, 2) deriving person-level variables from complex longitudinal within-person data, and 3) deriving variables that capture the latent structure of high dimensional data. The most useful techniques for feature engineering involve supervised learning, unsupervised learning, and the extraction of distribution parameters.

Supervised learning can be useful for extracting features from very granular or unstructured data. The term supervised learning refers to machine learning techniques that are used to predict values of a continuous (regression models), ordinal (ordinal regression) or discrete (multi-)nominal (classification models) target variable. For example, classification models are useful for inferring behaviors and events from multimodal sensing data (e.g., sleeping patterns, modes of transportation, or classes of physical activity). Similarly, object recognition algorithms can be used to extract features from image data (e.g., the presence of people, distinct facial expressions, or objects that are relevant to the research question at hand). Supervised learning models can be trained from scratch for the specific purpose of feature engineering but it is often more efficient to use pre-trained models. For example, object recognition can be performed with YOLO (Redmon et al., 2016) and abstract features can be extracted from image data using the convolutional bases of pre-trained convolutional neural network models available in the Keras library for $\mathrm{R}$ and Python (Chollet, 2015). Similarly, pre-trained word-embedding models can be used to derive features from natural language (e.g., Pennington et al., 2014). When dealing with longitudinal data, it can be helpful to fit time series regressions at the within-person-level and extract intercepts and slopes as between-person-level predictors capturing idiosyncratic behavioral trends over time. For example, researchers could use the slopes of within-person time series regressions to capture whether individuals increased (positive slope) or decreased (negative slope) their use of certain features of an academic app 
over time. These variables can then be used to predict other outcomes such as user satisfaction, attitudes towards features of the app, or academic performance measures.

Unsupervised learning is useful for identifying structure in complex data without explicitly modelling the relationships between predictors and a target variable. Cluster analysis can be useful to categorize data points based on similarity and dimensionality reduction techniques can help reduce the number of features by reducing the feature space to a smaller number of underlying dimensions. This process is especially useful when dealing with high dimensional data, such as text data. In the context of text data, other unsupervised techniques like topic modelling can be used for feature extraction, too. Topic modelling techniques, such as Latent Dirichlet Allocation (LDA; Blei et al., 2003), leverage latent structure in texts and identify themes that exist within a corpus of text based on the co-occurence of words across and within documents.

The simplest and most common feature engineering technique for longitudinal behavioral data is the extraction of distribution parameters. When presented with a time series of behaviors or events, researchers may be interested in their frequency and variability over time. Distribution descriptives like measures of central tendency and dispersion, entropy or skewness, and kurtosis are easy to extract and can provide a relatively good description of behavioral variation over time.

Of course, the three feature extraction strategies outlined above can be used in conjunction. It is often helpful to identify behaviors or behavioral clusters through supervised or unsupervised learning and then calculate summary statistics to aggregate these features to the person-level. In the analysis of GPS data, for example, unsupervised and supervised learning may be used to identify places where individuals tend to spend a lot of time (i.e., home, work, social places). Then, in a second step, researchers can analyze how people distribute their time across these different types of places to derive person-level individual difference measures that are related to person-level outcomes.

Finally, feature extraction is affected by researchers' decisions to engage in explanatory or predictive research. Explanatory research benefits from a low number of strong, highly interpretable features, whereas predictive research benefits from any feature that improves prediction performance. For this reason the feature engineering process in explanatory research is usually a top-down process, where researchers try to operationalize existing constructs. Feature engineering in predictive research, on the other hand, is often bottom-up, such that researchers first generate a multitude of atheoretical features and then try to optimize model performance through feature selection or shrinkage techniques. 


\section{Modelling}

Explanatory modelling and predictive modelling both benefit from an abundance of data because large datasets provide statistical power, safeguard against overfitting, and enable researchers to test their models out of sample without sacrificing precious training data. That is, large samples can be divided into sufficiently large subsamples some of which are used for model fitting while others are used to assess model performance on data that has not been previously used for modelling (e.g. through cross-validation; see below). On the other hand, big data poses unique challenges to the scalability of statistical methods and may require substantial computational resources. Generally, it is important to strike a tradeoff between the benefits of using more data and the corresponding increase in computational requirements. Needless to say, it is not always necessary to use the maximum amount of data available to draw scientific conclusions with a sufficient degree of confidence. For most psychological research questions, the difference between hundreds of thousands of data points and hundreds of millions of data points would be unlikely to fundamentally change the nature of the results. However, adding orders of magnitude of data can quickly increase computational requirements to a level where specialized hardware and software is needed or where the problem cannot be solved within a reasonable timeframe. The following sections discuss how explanatory and predictive modelling approaches depend on the structure of big data and explain common strategies to deal with big-N, big-V and big-T data.

\section{Statistical Inference for Big Data}

Statistical inference and the explanatory approach have their place in the world of big data, especially when big data is used to model causal mechanisms or to test theory-derived hypotheses, and when the assessment of uncertainty is a primary concern. In the field of psychology the dominant approach to statistical inference is frequentist and almost exclusively based on general linear models (GLMs), which include linear regression models, ANOVA, t-tests, and F-tests. Having access to large sample sizes has clear advantages in the explanatory framework. Most importantly, large sample sizes can help to prevent overfitting because larger samples represent the underlying population more accurately than smaller samples. Consequently, it is possible to fit more complex models without picking up on sample-specific patterns in the data, which do not exist in the broader population (Yarkoni \& Westfall, 2017). Similarly, even if large datasets are not representative, they can enable researchers to draw representative sub-samples or to focus on populations that are otherwise underrepresented in psychological research. For example, while Facebook users may on average be younger and better educated than the general population, the sheer size of datasets collected through Facebook provides access to sufficiently large numbers of individuals from underrepresented groups (Kosinski et al., 2015). 
However, the traditional statistical methods used by psychologists are not always well equipped to deal with large and complex datasets.

Traditional statistical analyses (i.e., GLMs) work reasonably well with big- $\mathrm{N}$ and some of the statistical assumptions such as the assumptions of normality of residuals or homoscedasticity may even be relaxed if sample size is sufficiently large (Maas \& Hox, 2004). However, sample size has obvious implications for significance testing and computation time. Specifically, large sample sizes make it easy to detect significant effects even when effect sizes are very small. Importantly, the types of real-world behavioral records that are characteristic of big-data are usually noisy and multi-determined, which imposes bounds on the effect-sizes that researchers can expect to find (Matz et al., 2017). With respect to computation, larger sample sizes entail longer execution times. For most of the common inference statistical methods runtime is a linear or quadratic function of sample size. In the rare case where execution time becomes prohibitive due to sample size, researchers can easily subsample their dataset and run the analysis on a sub-sample.

Big $\mathrm{V}$ data poses several unique problems for statistical inference methods. First, traditional statistical methods cannot computationally handle a large number of variables. Linear regression, for example, is computationally infeasible when the number of features exceeds the number of observations $(V>N)$ because there can be no unique coefficient estimate. Second, even when the number of variables does not exceed the number of observations $(V<N)$ linear regression may not scale well computationally because the relationship between the number of variables and runtime tends to be polynomial. Third, models become prone to overfitting with a growing number of variables. Fourth, models with a large number of variables are prone to multicollinearity, which causes imprecise coefficient estimates and inflated $p$-values. The last two points can, to some extent, be addressed by using regularized models (at the expense of biased coefficient estimates), dimensionality reduction (at the expense of interpretability), and variable selection (at the expense of potentially discarding relevant information).

Big T data requires special attention to the various potential sources of temporal effects beyond the effects that a researcher may be interested in. Modeling temporal data is perhaps the most difficult modeling scenario because there are various types of overlapping temporal effects (e.g., day of week, month of year, holiday, etc) that can easily distort interpretations. Moreover, there are additional complications with the transformation of time into a discrete measure. When an effect is small, distortions from suboptimal transformations can overwhelm the real signal by improperly weighing information. With respect to statistical analyses, techniques such as growth curve models, multi-level models and autoregressive models, can either make a specific temporal trend the focal point in an analysis or alternatively account for some temporal effects while focusing on more general patterns. In other cases it 
may be sufficient to extract person-level metrics that capture the properties of the underlying time series and model relationships at the person-level as in two stage regression (Gelman, 2005).

\section{Predictive Modelling for Big Data}

Predictive modelling is synonymous with supervised machine learning, where a target metric is predicted from a set of features. There are a variety of algorithms specifically designed for predictive modelling. These models range from regularized linear and logistic regression models (e.g., Tibshihani, 1996) to support vector machines (e.g., Cortes \& Vapnik, 1995), random forests (Breiman, 2001), gradient boosting (Friedman, 2000), and neural networks (for an introduction see Hastie et al., 2016). The algorithms used for predictive modelling are in some cases identical to the algorithms used for explanatory modelling, but the focus of the two approaches is fundamentally different. In contrast to explanatory modelling and statistical inference, predictive modelling does not place much emphasis on theoretical relationships represented by model coefficients, but instead emphasizes prediction performance. In place of significance tests and goodness-of-fit measures, predictive modelling requires the empirical assessment of generalizability by evaluating the model's prediction performance on new data; this is typically achieved through resampling and cross-validation (e.g., Stone, 1974).

Cross-validation in its most basic form describes the practice of randomly splitting a dataset into two subsets, one of which is used to train a model (training set), while the other one is used to assess prediction performance on data that has not been previously used to fit the model (testing set). Subsequently, the roles of the two datasets are reversed, such that the subset that has been previously used for testing is now used for training, while the subset that has been previously used for training is now used for testing. K-fold cross validation generalizes this process by repeating it with $\mathrm{k}$ splits, such that the model is trained on k-1 subsets and tested on the remaining subset in each iteration. This is repeated $\mathrm{k}$ times until each data point in the original dataset has been used for training and testing. Model performance is then assessed as the average prediction performance over all $k$ testing sets.

There are many variations to the cross validation approach. Leave-one-out cross-validation maximizes the size of the training set by performing $\mathrm{n}$ splits and repeatedly testing the model on a testing set with a sample size of one (e.g. Lachenbruch and Mickey, 1968). Monte-Carlo cross validation does not use mutually exclusive splits but draws a random subsample as a training set in each iteration; this has the advantage that the number of iterations becomes independent of the proportion of the data that is used for training and testing in each iteration (Xu \& Liang, 2001). Finally, there is nested cross-validation, which performs one or more additional splits within each training set. The inner-loop cross-validation cycle 
(looping through splits within the training set) is used for hyperparameter tuning (i.e., finding model configurations with high predictive power) while the outer loop (looping through train-test splits) is used to assess generalized model performance of the best models on testing data.

Hyperparameters are parameter values that can be specified by researchers to influence how a model learns (i.e., to control overfitting and learning speed). Hyperparameter tuning is an important step in predictive modelling because the hyperparameter configuration of an algorithm can have great impact on predictive performance. In the case of LASSO regression (Tibshirani, 1996), for instance, there is an additional regularization parameter (typically denoted as alpha or lambda), which determines how heavily coefficient values are penalized in the optimization process. In contrast to OLS regression, the LASSO algorithm does not only minimize the residual sum of squares but also the sum of the regression coefficients weighted by alpha. This ensures that the coefficients of unimportant predictors are set to zero, effectively removing these predictors from the model. By choosing the value of alpha researchers can determine how heavily coefficients are penalized. Choosing a high alpha value will emphasize penalization relative to the impact of squared residuals. Choosing a low alpha value will emphasize squared residuals. Because the level of penalization can greatly affect generalization performance, it is advisable to search a range of alpha values and pick the one that leads to the best results on new data. Other models like random forests, gradient boosting, and neural networks have a much higher number of hyperparameters that can be tuned. In these cases, hyperparameter configurations constitute a multidimensional search space. Multiple techniques have been suggested to optimize hyperparameter search, ranging from very simple options like grid search or random search (Bergstra \& Bengio, 2012) to more sophisticated approaches like Hyperband (Li et al., 2016) or bayesian optimization (Hutter et al., 2011), where additional machine learning models are used to optimize the parameters of the focal model.

The predictive approach and the cross-validation framework profit from big $\mathrm{N}$ data because it enables researchers to use hold-out sets for hyperparameter tuning and performance assessment without sacrificing training data. K-fold cross-validation can considerably increase computational requirements because each model needs to be fitted multiple (k) times. In a big $N$ context, however, it is easy to generate very large training sets and very large testing sets, which allows for a precise assessment of generalization performance without the need for a high number of cross-validation iterations. In addition to the fact that big $\mathrm{N}$ data facilitates hyperparameter search and the assessment of generalization performance, some of the most popular supervised learning algorithms are data hungry and require large training sets (e.g., neural networks).

Predictive modelling is usually not concerned with the interpretation of coefficients, so researchers can engage in feature engineering to produce large feature spaces and maximize prediction 
performance. At the same time, dimensionality reduction, regularization, and variable selection techniques are effective tools to tackle overfitting, which is a common problem with respect to big $V$ data. Dimensionality reduction techniques like matrix factorization, UMAP (McInnes et al., 2018), or autoencoder models (Rumelhart et al., 1986) can reduce the feature space to a smaller number of dimensions while retaining most of the information captured in the original feature space. A potential disadvantage of these techniques is the reduced interpretability of the new features. Regularized linear models like LASSO, ridge, or elastic-net regressions have a specific hyperparameter (alpha), which controls how heavily model coefficients are penalized. In heavily regularized models (high alpha), only the features with the highest predictive power will have non-zero coefficients, while features that have a negligible impact on model fit will be filtered out. This reduces model complexity and therefore counteracts overfitting.

Model complexity can also be reduced through model-based and model-agnostic feature selection techniques. Model-based feature selection works with feature importance metrics derived from a fitted model, such as the magnitude of coefficients in linear models or impurity decrease in random forest models. A common technique is recursive model-based selection, where a model is repeatedly fitted while the feature with the lowest importance score is discarded in each iteration. Model-agnostic feature selection techniques, on the other hand, do not rely on feature importance metrics derived from the model itself, but they observe how prediction performance reacts to changes in the feature space. Permutation importance, for instance, permutes the values of a feature to eliminate all associations between feature values and target values. The subsequent reduction in prediction performance quantifies the importance of the focal feature. Based on permutation importance, features can be selected in a forward (select most important features first) or backward (eliminate least important features first) manner. Feature selection algorithms usually rely on fitting the model repeatedly so their computational costs can be prohibitive in big data contexts.

As mentioned above, inference statistical models can be used for predictive modelling in conjunction with cross validation, but can machine learning methods be used for explanation rather than prediction? Research concerned with model interpretability and model explainability has made tremendous progress in recent years and produced many methodological innovations enabling researchers to better understand the predictions made by black-box models. However, being able to explain predictions of a model is not the same as being able to explain the underlying data generating process or causal mechanisms. Feature importance scores derived from machine learning models, for example, are fundamentally different from coefficient estimates in statistical models. Feature importance scores, such as permutation importances, are generally nonlinear. In the case of kernel SVMs, random forest models, gradient boosting models, and neural networks, feature importance scores capture 
polynomial relationships and complex interactions. It is therefore difficult to infer directionality or interpret effects in isolation from the effects of other features. Similarly, in the case of penalized regression models, the coefficient estimates are biased because the objective function minimizes the combined sum of squared residuals and coefficient values. Coefficients therefore depend on the regularization parameter set by the researcher and in the presence of correlated features the algorithm arbitrarily removes some of them, rendering the remaining coefficients uninterpretable.

Nonetheless, as pointed out earlier, there are at least three major ways in which predictive modelling can support explanatory research (Shmueli, 2010). First, predictive modelling can uncover new phenomena and causal mechanisms by taking into account complex data and a multitude of potential relationships. Second, predictive modelling can be used to discover new measures and operationalizations through bottom-up feature engineering. Dimensionality reduction, regularization, and variable selection techniques can be used to find meaningful predictive relationships in rich feature spaces, which can then be integrated into existing theory or serve as starting points for theoretical advancement. In practice that means that researchers would use simple explanatory models to better understand the findings uncovered by complex predictive models. Third, predictive modelling can serve as a reality check for explanatory models. In practice this means that theoretical findings produced by explanatory modelling can be subsequently assessed with regard to their prediction performance using the cross-validation approach. We recommend that researchers routinely validate the generalizability of inference statistical findings by means of cross validation. Finally, recent research suggests that algorithms traditionally associated with predictive the approach can be used for causal inference and the estimation of treatment effects (e.g. Farrell et al., 2021; Shi et al., 2019; Steinkraus, 2019).

\section{Resource Considerations}

As noted earlier, the prospect of running a computational procedure in an acceptable amount of time depends on the difficulty of the computational problem and the computational resources available. Computational resources in turn depend on the hardware setup, most importantly memory capacity and processing power. It is therefore helpful for researchers to assess how preprocessing and modelling procedures scale with respect to computational requirements.

\section{Computational Complexity}


In the field of computer science, computational complexity theory is chiefly concerned with the classification of algorithms according to their resource requirements. Psychologists certainly do not need to become experts in this highly theoretical area of research, but developing a basic intuition of computational complexity can help avoid certain pitfalls when working with large datasets.

In general, the computational requirements of an algorithm grow with the size of the input data. The relationship between input size and computational requirements can be characterized by identifying the order of the corresponding growth function (e.g., linear, polynomial, exponential, factorial). In the context of data analysis it is usually most important to assess how preprocessing and modelling procedures scale with respect to sample size and the number of variables. This relationship can be expressed using the "Big O notation", where $O$ denotes the order of the function. Assuming that a dataset is represented as an input matrix with the dimensions $n$ (sample size) and $p$ (number of variables) runtime can be expressed as a function of $n$ and $p$. A linear relationship between sample size and runtime, for example, can be expressed as $O(n)$, whereas a polynomial relationship of degree 3 between the number of variables and runtime would be expressed as $\mathrm{O}\left(\mathrm{p}^{3}\right)$. The complexity of most procedures depends on both $\mathrm{n}$ and $\mathrm{p}$, so most expressions contain both dimensions. The runtime for least squares fitting, for instance, would be $\mathrm{O}\left(\mathrm{p}^{3}+\mathrm{n} \mathrm{p}^{2} / 2\right)$ or $\mathrm{O}\left(n \mathrm{p}^{2}\right)$ depending on the choice of the decomposition algorithm (see Hastie et al., 2016). That means that computational costs for linear regression models are especially sensitive to the number of variables involved. Importantly, the same computational goal can often be achieved through different algorithms with different computational complexities. Knowing the runtime complexity of a given algorithm can give researchers an idea of how the algorithm scales and may therefore affect their decisions regarding the analytic strategy, the hardware configuration that is needed, and how much data should be used. The computational complexity of an algorithm is usually reported in the publication introducing the algorithm. For common algorithms computational considerations are discussed in statistics and computer science textbooks such as Elements of Statistical Learning (Hastie et al., 2016). Additionally, because many techniques like hyperparameter tuning, cross-validation, or feature selection rely on fitting models repeatedly, it is advisable for researchers to first run a single model and then extrapolate execution time to the overall learning task.

\section{Processing Power}

Computational operations are usually performed by the CPU. Other things equal, a faster CPU (or in the case of parallel processing a CPU with more cores) can perform more operations on data and will therefore improve the speed with which the data are processed. As the computational requirements for big data applications are generally very high, differences in processing power and parallelization can 
lead to appreciable differences in execution time. The term parallelization or parallel computing describes a paradigm where a computational task is divided into subtasks that can be run independently and simultaneously on different CPUs or CPU cores. The performance gain that can be achieved through parallelization depends not only on the hardware but also on properties of the computational task at hand. Highly parallelizable tasks can easily be split up into independent sub-tasks, whereas tasks that have a lot of interdependencies are harder to parallelize. Examples of easily parallelizable tasks in the context of machine learning are tasks that rely on repeated execution of subtasks, such as hyperparameter tuning, cross-validation, and variable selection, or ensemble models like random forests, where many base estimators are fitted independently. Tasks that are hard to parallelize include iterative processes where each computational subtask relies on the results of previous subtasks. This is the case for sequential methods, such as Markov Chain Monte Carlo (MCMC) algorithms, which play an important role in Bayesian machine learning. Both Python and R run on a single CPU core by default, which is often insufficient when large quantities of data need to be processed. However, standard machine learning software usually offers some multi-core capabilities (e.g., $n \_j o b s$ Scikit-Learn). Additionally, both languages provide libraries that facilitate parallel computing (e.g., doParallel and foreach in R; Dask and concurrent in Python) and enable users to distribute tasks across multiple cores. Using multiple cores on a local machine is certainly preferable to running code on a single core while the other cores are idle, but truly large datasets may require more processing power than any laptop or desktop computer can provide. In these cases it is advisable to use cloud computing and run code on a powerful server or on a high-performance computing cluster (HPC). A cluster is a set of servers (i.e., nodes) that works together to tackle a computational task. Researchers can flexibly determine the structure and the size of a cluster when using cloud services like Amazon Web Services, Google Cloud Platform, or Microsoft Azure. To make efficient use of a cluster, special software such as Apache Spark is required. Spark offers data manipulation and machine learning libraries optimized for parallelized distributed computing. Both $\mathrm{R}$ and Python can be used to interact with the Spark API. An additional advantage of using cloud services is that researchers can opt for specialized hardware such as graphics processing units (GPUs) or tensor processing units (TPUs), which are optimized for machine learning tasks, especially in the context of deep learning.

\section{Memory}

Memory is used to temporarily store data for quick access so that the CPU can perform computations on them. In most standard applications the data that are being analyzed are fully loaded into memory. While the memory capacity of laptops and desktop machines has dramatically increased over the past decades, big data will easily push the memory of standalone machines to its limits. In such 
cases it is advisable to resort to more powerful remote machines or distributed computing. But even if memory can easily be expanded, as in cloud computing, researchers should be aware of how they use memory, because inefficient memory use can considerably slow down data processing. First, it is advisable to drop unnecessary data early on in the data analysis process. Second, researchers should avoid creating unnecessary copies of the data they are working with. A common mistake is the creation of copies when performing operations in a loop. Third, objects that are not needed anymore can be deleted from memory. Fourth, it is important to pay close attention to data structures and data types that are being used because different data structures and data types may have different memory requirements. For example, the use of the sparse matrix format can improve memory efficiency. Related to this point, researchers should be aware of how the transformations that they apply to the data interact with data structures and data types. Naively standardizing a sparse matrix, for instance, can dramatically increase memory requirements by replacing zeros with non-zero values.

If memory is still an issue after optimizing the code for memory efficiency and buying more memory or renting cloud memory is not an option, researchers can resort to several workarounds. First, it is possible to use secondary storage as "virtual memory". In practice, that means that disc space is treated like memory, which effectively expands memory capacity but will slow down operations that are performed on data that is stored on the disc. Second, it is often possible to process data in chunks, such that only a subset of data (a chunk) is loaded into memory. Once the data is processed, it is released from memory, so that the next chunk can be processed. The results of the operations performed on each of the chunks can then be assembled for further processing. This idea can be illustrated in the context of text analysis, where individual documents or groups of documents can be processed separately and derived metrics like word counts can be appended to a results file. Both $\mathrm{R}$ and Python offer tools that can help to overcome memory limitations. The $\mathrm{R}$ packages ff, filehash or disc.frame may be a reasonable starting point. In Python the popular pandas library enables researchers to read and process files in chunks. However, as mentioned above, it is advisable to resort to cloud solutions and rent additional resources if memory capacity is a limiting factor.

\section{Conclusion}

Big data opens up a broad range of interesting new research opportunities for psychologists. Here we have discussed some of the opportunities and challenges associated with different kinds of big data (big N, big V, big $\mathrm{T}$ ) for psychological research. Furthermore, we have assembled a collection of current best practices for data collection (APIs, web-scraping, industry collaborations), data storage (RDBMSs, distributed file systems, cloud storage), preprocessing (filtering and merging, missing and 
sparse data, feature engineering) and modelling (explanatory and predictive) of large, complex data. While the current chapter is certainly too short to cover the intricacies of big data research it was our goal to introduce the reader to the most important concepts and provide starting points for further reading. We hope that in doing so this chapter will facilitate innovative work using big data in the field of psychology 
Adjerid, I., \& Kelley, K. (2018). Big data in psychology: A framework for research advancement. American Psychologist, 73(7), 899-917. https://doi.org/10.1037/amp0000190

Bergstra, J., \& Bengio, Y. (2012). Random Search for Hyper-Parameter Optimization. The Journal of Machine Learning Research, 13, 281-305.

Blei, D. M., Ng, A. Y., \& Jordan, M. I. (2003). Latent Dirichlet Allocation. The Journal of Machine Learning Research, 3(null), 993-1022.

Borgman, C. L. (2015). Big Data, Little Data, No Data: Scholarship in the Networked World. The MIT Press; JSTOR. https://doi.org/10.2307/j.ctt17kk8n8

Breiman, L. (2001). Random Forests. Machine Learning, 45(1), 5-32. https://doi.org/10.1023/A:1010933404324

Camerer, C. F., Dreber, A., Holzmeister, F., Ho, T.-H., Huber, J., Johannesson, M., Kirchler, M., Nave, G., Nosek, B. A., Pfeiffer, T., Altmejd, A., Buttrick, N., Chan, T., Chen, Y., Forsell, E., Gampa, A., Heikensten, E., Hummer, L., Imai, T., ... Wu, H. (2018). Evaluating the replicability of social science experiments in Nature and Science between 2010 and 2015. Nature Human Behaviour, 2(9), 637-644. https://doi.org/10.1038/s41562-018-0399-z

Chollet, F. (2015). Keras: The Python deep learning API. https://keras.io/

Cortes, C., \& Vapnik, V. (1995). Support-vector networks. Machine Learning, 20(3), 273-297. https://doi.org/10.1007/BF00994018

Ebert, T., Götz, F. M., Obschonka, M., Zmigrod, L., \& Rentfrow, P. J. (2019). Regional variation in courage and entrepreneurship: The contrasting role of courage for the emergence and survival of start-ups in the United States. Journal of Personality, 87(5), 1039-1055. https://doi.org/10.1111/jopy.12454

Enamorado, T., Fifield, B., \& Imai, K. (2019). Using a Probabilistic Model to Assist Merging of Large-Scale Administrative Records. American Political Science Review, 113(2), 353-371. https://doi.org/10.1017/S0003055418000783

Farrell, M. H., Liang, T., \& Misra, S. (2021). Deep Neural Networks for Estimation and Inference. Econometrica, 89(1), 181-213. https://doi.org/10.3982/ECTA16901

Friedman, J. H. (2000). Greedy Function Approximation: A Gradient Boosting Machine. Annals of 
Statistics, 29, 1189-1232.

Gelman, A. (2005). Two-Stage Regression and Multilevel Modeling: A Commentary. Political Analysis, 13(4), 459-461. https://doi.org/10.1093/pan/mpi032

Gosling, S. D., Vazire, S., Srivastava, S., \& John, O. P. (2004). Should We Trust Web-Based Studies? A Comparative Analysis of Six Preconceptions About Internet Questionnaires. American Psychologist, 59(2), 93. https://doi.org/10.1037/0003-066X.59.2.93

Hastie, T., Tibshirani, R., \& Friedman, J. (2016). The Elements of Statistical Learning: Data Mining, Inference, and Prediction, Second Edition (2nd edition). Springer.

Hutter, F., Hoos, H. H., \& Leyton-Brown, K. (2011). Sequential Model-Based Optimization for General Algorithm Configuration. In C. A. C. Coello (Ed.), Learning and Intelligent Optimization (pp. 507-523). Springer Berlin Heidelberg.

Isaak, J., \& Hanna, M. J. (2018). User Data Privacy: Facebook, Cambridge Analytica, and Privacy Protection. Computer, 51(8), 56-59. https://doi.org/10.1109/MC.2018.3191268

Kosinski, M., Matz, S. C., Gosling, S. D., Popov, V., \& Stillwell, D. (2015). Facebook as a research tool for the social sciences: Opportunities, challenges, ethical considerations, and practical guidelines. American Psychologist, 70(6), 543-556. https://doi.org/10.1037/a0039210

Kosinski, M., Stillwell, D., \& Graepel, T. (2013). Private traits and attributes are predictable from digital records of human behavior. Proceedings of the National Academy of Sciences, 110(15), 5802-5805. https://doi.org/10.1073/pnas.1218772110

Kulkarni, V., Kern, M. L., Stillwell, D., Kosinski, M., Matz, S., Ungar, L., Skiena, S., \& Schwartz, H. A. (2018). Latent human traits in the language of social media: An open-vocabulary approach. PLOS ONE, 13(11), e0201703. https://doi.org/10.1371/journal.pone.0201703

Lachenbruch, P. A., \& Mickey, M. R. (1968). Estimation of Error Rates in Discriminant Analysis. Technometrics, 10(1), 1-11. https://doi.org/10.1080/00401706.1968.10490530

Li, L., Jamieson, K., DeSalvo, G., Rostamizadeh, A., \& Talwalkar, A. (2016). Hyperband: A Novel Bandit-Based Approach to Hyperparameter Optimization. ArXiv:1603.06560 [Cs, Stat]. http://arxiv.org/abs/1603.06560 
Maas, C. J. M., \& Hox, J. J. (2004). The influence of violations of assumptions on multilevel parameter estimates and their standard errors. Computational Statistics \& Data Analysis, 46(3), 427-440. https://doi.org/10.1016/j.csda.2003.08.006

Matz, S. C., Gladstone, J. J., \& Stillwell, D. (2017). In a World of Big Data, Small Effects Can Still Matter: A Reply to Boyce, Daly, Hounkpatin, and Wood (2017). Psychological Science, 28(4), 547-550. https://doi.org/10.1177/0956797617697445

McInnes, L., Healy, J., \& Melville, J. (2018). UMAP: Uniform Manifold Approximation and Projection for Dimension Reduction. ArXiv:1802.03426 [Cs, Stat]. http://arxiv.org/abs/1802.03426

Mueller, S., Peters, H., Matz, S., Wang, W., \& Harari, G. (in Press). Investigating the Relationships Between Mobility Behaviors and Indicators of Subjective Well-Being Using Smartphone-based Experience Sampling and GPS Tracking. European Journal of Personality.

Open Science Collaboration. (2015). Estimating the reproducibility of psychological science. Science, 349(6251), aac4716-aac4716. https://doi.org/10.1126/science.aac4716

Pennington, J., Socher, R., \& Manning, C. (2014). Glove: Global Vectors for Word Representation. Proceedings of the 2014 Conference on Empirical Methods in Natural Language Processing (EMNLP), 1532-1543. https://doi.org/10.3115/v1/D14-1162

Redmon, J., Divvala, S., Girshick, R., \& Farhadi, A. (2016). You Only Look Once: Unified, Real-Time Object Detection. 2016 IEEE Conference on Computer Vision and Pattern Recognition (CVPR), 779-788. https://doi.org/10.1109/CVPR.2016.91

Rentfrow, P. J., Jokela, M., \& Lamb, M. E. (2015). Regional Personality Differences in Great Britain. PLOS ONE, 10(3), e0122245. https://doi.org/10.1371/journal.pone.0122245

Rumelhart, D. E., Hinton, G. E., \& Williams, R. J. (1986). Learning representations by back-propagating errors. Nature, 323(6088), 533-536. https://doi.org/10.1038/323533a0

Schneble, C. O., Elger, B. S., \& Shaw, D. (2018). The Cambridge Analytica affair and Internet-mediated research. EMBO Reports, 19(8). https://doi.org/10.15252/embr.201846579

Shi, C., Blei, D., \& Veitch, V. (2019). Adapting Neural Networks for the Estimation of Treatment Effects. 33rd Conference on Neural Information Processing Systems (NeurIPS 2019), Vancouver, 
Canada.

Shmueli, G. (2010). To Explain or to Predict? Statistical Science, 25(3), 289-310.

https://doi.org/10.1214/10-STS330

Stachl, C., Pargent, F., Hilbert, S., Harari, G. M., Schoedel, R., Vaid, S., Gosling, S. D., \& Bühner, M. (2020). Personality Research and Assessment in the Era of Machine Learning. European Journal of Personality, 34(5), 613-631. https://doi.org/10.1002/per.2257

Steinkraus, A. (2019). Estimating Treatment Effects With Artificial Neural Nets-A Comparison to Synthetic Control Method. Economics Bulletin, 39(4), 2778-2791.

Stone, M. (1974). Cross-Validatory Choice and Assessment of Statistical Predictions. Journal of the Royal Statistical Society. Series B (Methodological), 36(2), 111-147.

Tibshirani, R. (1996). Regression Shrinkage and Selection via the Lasso. Journal of the Royal Statistical Society. Series B (Methodological), 58(1), 267-288.

Varol, O., Ferrara, E., Davis, C. A., Menczer, F., \& Flammini, A. (2017). Online Human-Bot Interactions:

Detection, Estimation, and Characterization. ArXiv:1703.03107 [Cs].

http://arxiv.org/abs/1703.03107

Xu, Q.-S., \& Liang, Y.-Z. (2001). Monte Carlo cross validation. Chemometrics and Intelligent Laboratory Systems, 56(1), 1-11. https://doi.org/10.1016/S0169-7439(00)00122-2

Yarkoni, T., \& Westfall, J. (2017). Choosing Prediction Over Explanation in Psychology: Lessons From Machine Learning. Perspectives on Psychological Science, 12(6), 1100-1122. https://doi.org/10.1177/1745691617693393

Youyou, W., Kosinski, M., \& Stillwell, D. (2015). Computer-based personality judgments are more accurate than those made by humans. Proceedings of the National Academy of Sciences, 112(4), 1036-1040. https://doi.org/10.1073/pnas.1418680112 